\title{
Telangiectasia macularis eruptive perstans under the dermoscope in the skin of color
}

\section{Subrata Malakar', Samipa Samir Mukherjee ${ }^{2}$}

\author{
${ }^{1}$ Department of Dermatology, Rita Skin Foundation, Kolkata, India, ${ }^{2}$ Department of Pediatric Dermatology, Cloudnine \\ Hospitals, Bengaluru, India
}

Corresponding author: Dr. Samipa Samir Mukherjee, E-mail: drsamipamukherjee@gmail.com

Sir,

Cutaneous mastocytosis is characterized by the proliferation and accumulation of mast cells in the skin and is characterized by five subtypes including urticarial pigmentosa, diffuse cutaneous mastocytosis, bullous mastocytosis, solitary mastocytoma and Telangiectasia macularis eruptive perstans (TMEP). TMEP is a rare disease, found in less than $1 \%$ of patients with cutaneous mastocytosis [1]. It affects predominantly adults and is characterized by erythematous and/ or yellow-brownish macules with telangiectasias, preferably located on the trunk and upper limbs. Darier sign (urticaria after the friction of a lesion) is absent in most cases. Dermoscopy reveals a characteristic vascular pattern in TMEP thereby aiding in diagnosis.

A 34 year-old male patient presented with erythematous skin lesions on the upper chest, back and upper limbs of 3 years duration (Figs. 1 and 2). He denied any itching in the lesions. Cutaneous examination revealed erythematous-brownish macules ranging from 0.5 to $5.0 \mathrm{~cm}$ in diameter, confluent, distributed in the chest, back and upper limbs, and few residual hyperchromic macules. Darier sign was negative. History and systemic examination was not contributory. Dermoscopy of the erythematous-brownish lesions revealed thin and tortuous linear vessels in a reticular pattern, yellow brown background, mild erythema and delicate pigment network (Fig. 3). Histopathological examination showed dilated vessels with moderate inflammatory reaction around, mainly composed of mast cells, best visualized with Giemsa stain. Thus the diagnosis of TMEP was established.

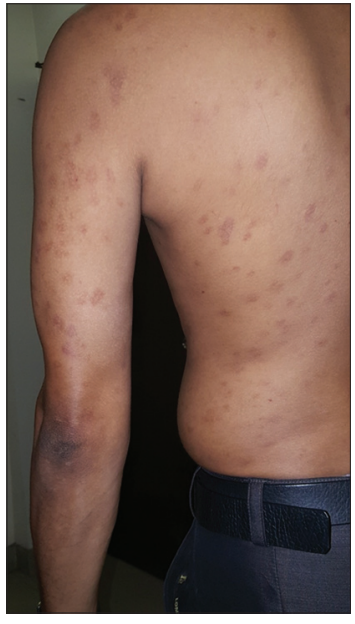

Figure 1: Erythematous brownish macules on the back and arm.

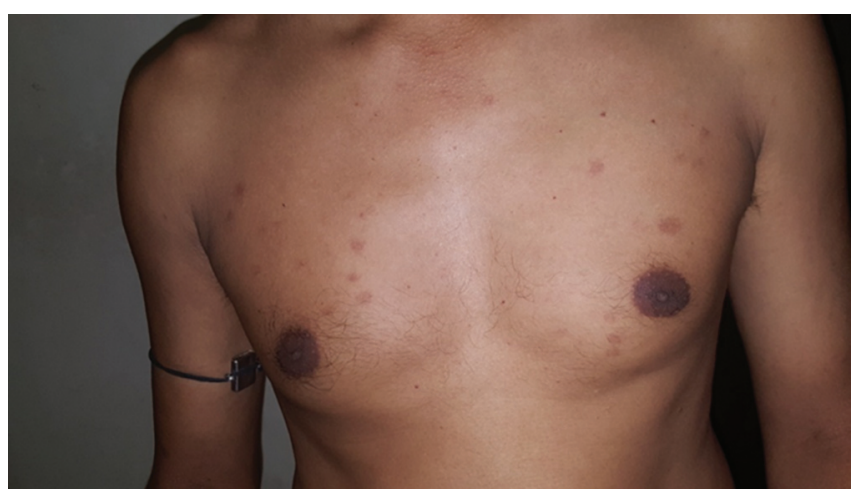

Figure 2: Erythematous brownish macules on the upper chest.

In 2009, Akay et al studied the dermatoscopic pattern in 6 patients with different forms of cutaneous mastocytosis and described the pigmented network and reticular vascular pattern. The pigmented network was observed predominantly in patients with maculopapular mastocytosis and urticaria pigmentosa and the reticular vascular pattern in patients with TMEP [2].

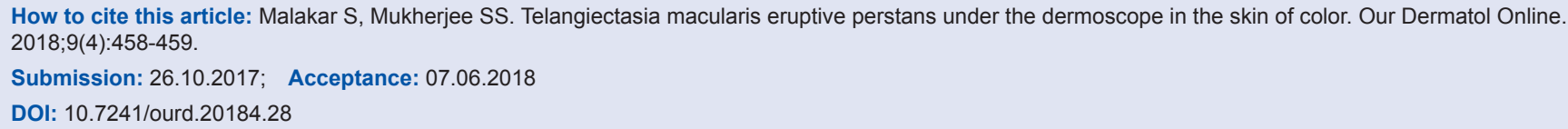




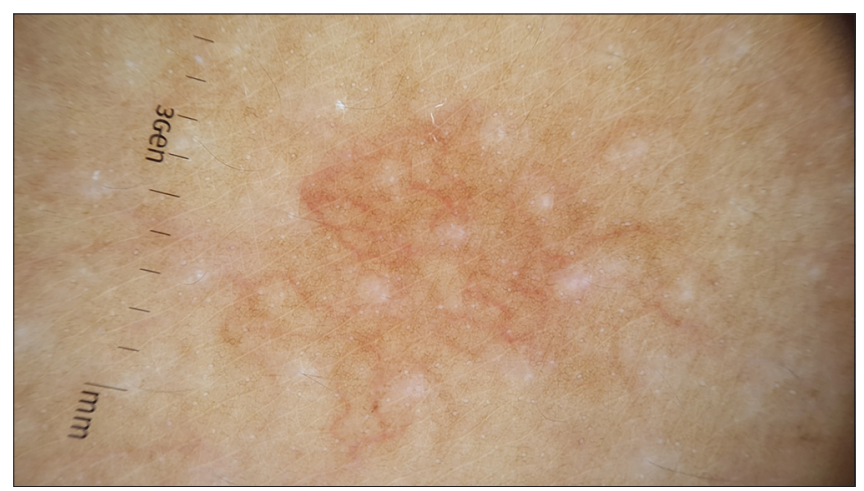

Figure 3: Mild erythema, thin and tortuous linear vessels, delicate pigment network, yellow-brown background.

Further in 2011,Vano-Galvan et al evaluated the dermoscopic findings of 127 patients with cutaneous mastocytosis and characterized four distinct patterns comprising of pigmented network, yellow-orange amorphous area, brown amorphous area and telangiectasia with reticular pattern [3].

In 2013 Unterstell et al reported similar dermoscopic findings of thin and tortuous linear vessels, mild erythema and fine pigment network in their report [4].

The Dermoscopic findings of the characteristic reticular vascular pattern, corresponds to dilatation and vascular proliferation associated with the presence of mast cells in the dermis. The mild erythema reported on dermoscopy reported in few cases in the literature may be difficult to appreciate in the skin of colour.

To the best of our knowledge this is the first report of dermoscopic findings in TMEP from the Indian subcontinent and we believe that dermoscopy can serve as an important adjunct in the effective diagnosis of this entity.

\section{Consent}

The examination of the patient was conducted according to the Declaration of Helsinki principles.

\section{REFERENCES}

1. Carvalho IM, Friedman H, Medeiros BM. Telangiectasia macularis etuptiva perstans: a case report. An Bras Dermatol. 2001;76:611-4.

2. Akay BN, Kittler H, Sanli H, Harmankaya K, Anadolu R. Dermatoscopic findings of cutaneous mastocytosis. Dermatology. 2009;218:226-30.

3. Vano-Galvan S, Alvarez-Twose I, De las Heras E, Morgado JM, Matito A, Sánchez-Muñoz L, et al. Dermoscopic features of skin lesions in patients with mastocytosis. Arch Dermatol. 2011;147:932-40.

4. Unterstell N, Lavorato FG, Nery NS, Mann D, Alves M de FSG, Barcauí C. Dermatoscopic findings in telangiectasia macularis eruptiva perstans. Anais Bras Dermatol. 2013;88:643-5.

Copyright by Subrata Malakar, et al. This is an open-access article distributed under the terms of the Creative Commons Attribution License, which permits unrestricted use, distribution, and reproduction in any medium, provided the original author and source are credited.

Source of Support: Nil, Conflict of Interest: None declared. 